\title{
The Practice of Intensive Nursing Skill Training in Clinical Nursing Teaching
}

\author{
Si Xuyan \\ Department of Clinical Basic Medicine, Zhengzhou University of Industrial Technology, Zhengzhou \\ China
}

\begin{abstract}
Keywords: Intensive nursing skills; Nursing education; Practice
Abstract. To investigate the effect of intensive nursing skills in clinical teaching. Method Selecting 60 students of nursing in our school, randomly divided into control group (30 cases) and observation group (30 cases). The control group uses routine nursing skills, and the observation group uses intensive nursing skills. Then compare the scores of OSCE, the score of clinical comprehensive ability and the total satisfaction of training methods between the two groups. Result Two groups of students' scores of OSCE had no significant difference before training $(p>0.05)$, the OSCE assessment scores were significantly improved after training. OSCE scores comparison before training $(\mathrm{p}=0.7219, \mathrm{p}=0.3876, \mathrm{p}=0.9223, \mathrm{p}=0.2434)$; OSCE scores comparison after training ( $\mathrm{p}$ $=0.0221, \mathrm{p}=0.0328, \mathrm{p}=0.0000, \mathrm{p}=0.0000)$. Two groups of students' clinical comprehensive ability scores before training had no significant difference $(\mathrm{P}>0.05)$, after training the scores of clinical comprehensive ability has significantly improved. Clinical comprehensive ability comparison before training $(\mathrm{p}=0.3062, \mathrm{p}=0.9516, \mathrm{p}=0.4420, \mathrm{p}=0.7776)$; clinical comprehensive ability comparison after training $(\mathrm{p}=0.0332, \mathrm{p}=0.0374, \mathrm{p}=0.007, \mathrm{p}=0.0111)$. observation group's total satisfaction of intensive nursing methods $(90.00 \%)$ is higher than that of control group $(63.33 \%)$, the difference was statistically significant $(\mathrm{p}<0.05)$.
\end{abstract}

\section{Introduction}

Nursing is a strong practical application subject, it is an important bridge to achieve school teaching and clinical practice [1]. A decent nurse should not only master the nursing skills, but also have comprehensive ability such as teamwork, expression and communication ability, observation judgment, emergency response, health education [2]. Carrying out standardized nursing skills training is an important part before students' clinical practice, routine nursing skills training cannot meet the practical, complex type advanced nursing talents' requirements [3] so colleges and universities need to focus on the cultivation of the students' clinical practice comprehensive ability in clinical nursing teaching.

\section{Data and Methods}

General Information. Select 60 nursing students in our school who are going out to practice, divide them into control group and observation group according to the random number table method, 30 cases in each group. There are 9 males, 21 females in control group, aged 23 to 24 years, average age $(23.50 \pm 0.25)$; there are 6 males and 24 females in observation group, aged 22 to 24 years, average age $(23.00 \pm 0.85)$, the two groups of students are about to practice, all of them possess basic theoretical knowledge (basic nursing, medical-surgical nursing, pediatrics and obstetrics and gynecology nursing). Compare the two groups of students' baseline, there is no significant difference ( $p>0.05)$, which are comparable.

Methods. 2 groups of students' training content for the basic operation of nursing subjects includes 12 basic operation skills and comprehensive ability training such as aseptic technique, intravenous injection.

Control group: Use the routine nursing skills training, first divide into groups within control group, 10 for a group, develop training plans according to the training content and objectives, develop curriculum training programs according to the hours of teachers. In the process of training, teachers 
demonstrate with case, and then let the students within the group to carry out repeated exercises. In the process of practicing, teachers give guidance and correction and encourage students to practice after school; cultivate students' comprehensive ability like emergency response, observation and judgment during the training course.

Observation group: Use intensive nursing skills training. Training is divided into the following steps: (1) pre-training preparation: Before intensive training, the teacher will organize theoretical and operational knowledge of the training, write typical clinical cases in all the subjects, distribute data to students a week before the training (To exercise the comprehensive ability of students, exercise in group of five in observation group), so that students can find the case-related information based on case content, review of relevant theoretical knowledge independently. (2) Training: Training in groups in the laboratory, people in the group get division of labor to do simulated training immediately, teachers set certain emergency according to the case data to examine the students' emergency response and the ability to deal with problems, teachers give guidance during the process. After a round of training, the teacher guides students in the group to discussion, self-evaluation and mutual evaluation, pointing out the inadequacies or improvements, and finally the teacher gives a summary and demonstration of standardized operation, let students exchange roles in the second round of training until they master operating skills so far. (3) After training: After the end of intensive training, let students review the relevant theoretical knowledge to consolidated it, and encourage students to practice after school.

Evaluation index and judgment index. Evaluation: Based on objective structured clinical examination

(Objective Structured Clinical Examination, OSCE) evaluation[4] includes 4 stations, case analysis in all the subjects, nursing assessment, operating skills, health education examination, total score is 100 points in each station. Score of 90 points or more is divided into excellent, 80-89 is divided into good, $61-79$ is divided into medium, 60 points pass, 60 points below fail. Guide teachers evaluate clinical comprehensive ability of students after training, investigate teamwork, observation and judgment, emergency response, communication skills. Each item scores 10 points, excellent: 9 points or more; good: 8-9 points; 6-8 points: medium; The higher the score is, the stronger the overall comprehensive clinical capacity is.

Indices of Compatibility: According to the self - made "Questionnaire of Clinical Nursing Teaching Satisfaction in Different Training Methods" to do question investigation. Very satisfied: nursing skills, theoretical knowledge, comprehensive ability is consolidated and enhanced greatly; Satisfied: nursing skills, theoretical knowledge, comprehensive ability is consolidated and enhanced to a certain degree. General satisfied: nursing skills, theoretical knowledge, comprehensive ability is consolidated, but enhanced inadequately; Not satisfied: nursing skills, theoretical knowledge, comprehensive ability is practiced and consolidated, but there is no upgrade. Total Satisfaction= (very satisfied + satisfied) / total cases $\times 100 \%$.

Statistical analysis. All the resulting data of this study use SPSS16.0 for Windows for statistical analysis, measurement data use t-test, presenting with $(-\chi \pm \mathrm{s})$, counting data are examined by $\mathrm{u}$-test; the statistically significant difference is indicated with $\mathrm{p}<0.05$.

\section{The Results}

The OSCE assessment score of two groups of students. There was no significant difference $(p>0.05)$ in the OSCE score of two groups of students before training, after training all the scores have improved, score of the observation group was higher than that of control group, the comparison had differences $(\mathrm{p}<0.05)$, was statistically significant. Details as shown in table 1 
Table 1 The 0SCE assessment score of two groups of students before and after training [n, ($\mathrm{x} \pm \mathrm{s})$, points]

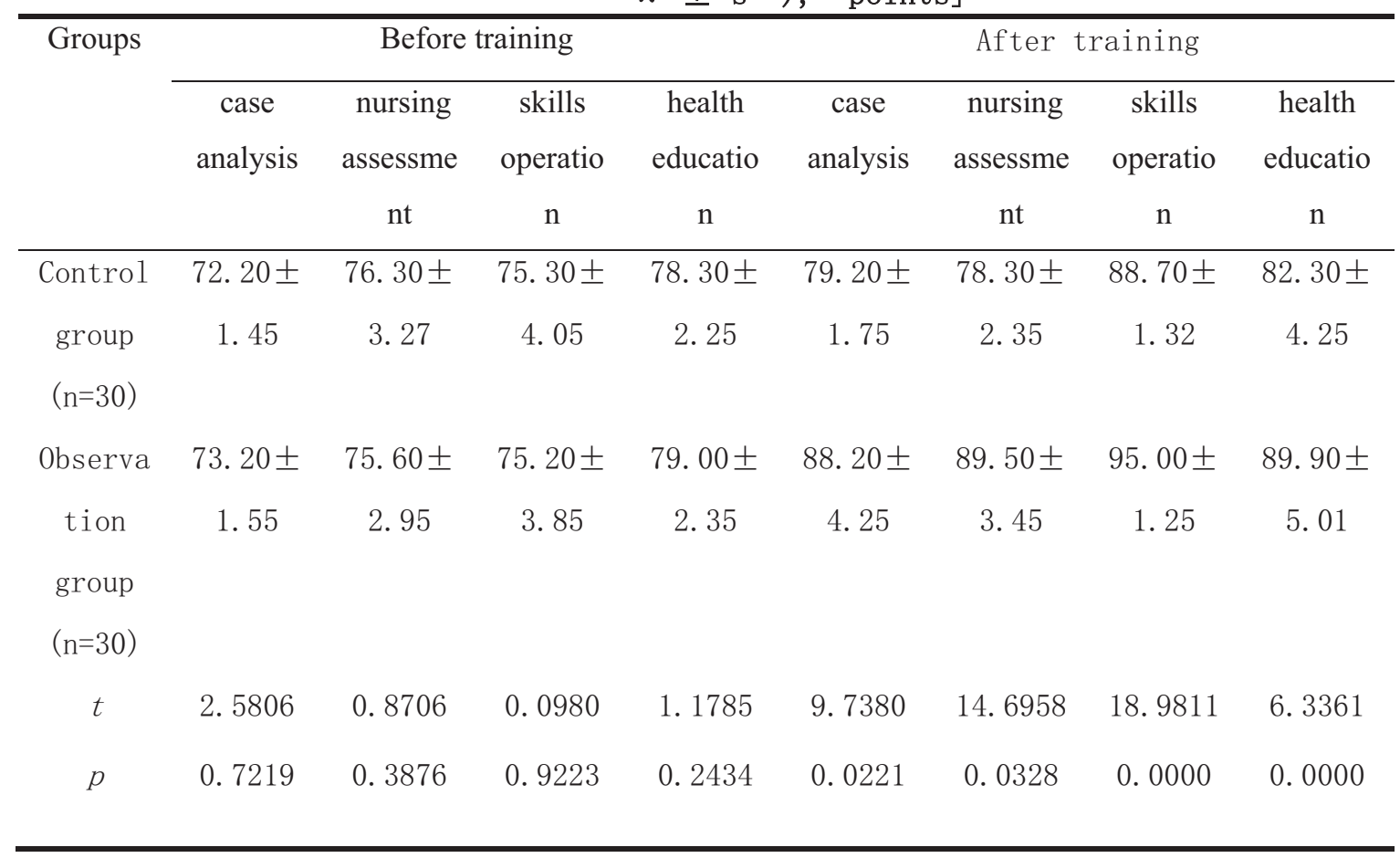

The clinical comprehensive ability score of two groups of students. Comprehensive ability score of two groups of students before training had no difference ( $p>0.05)$, but it has improved after training, the observation group's score was higher than the control group's, the comparison had differences $(\mathrm{p}<0.05)$, was statistically significant. Details as shown in table 2

Table 2 clinical comprehensive ability of two groups of students before and after training [n, $\overline{(} \chi \pm$

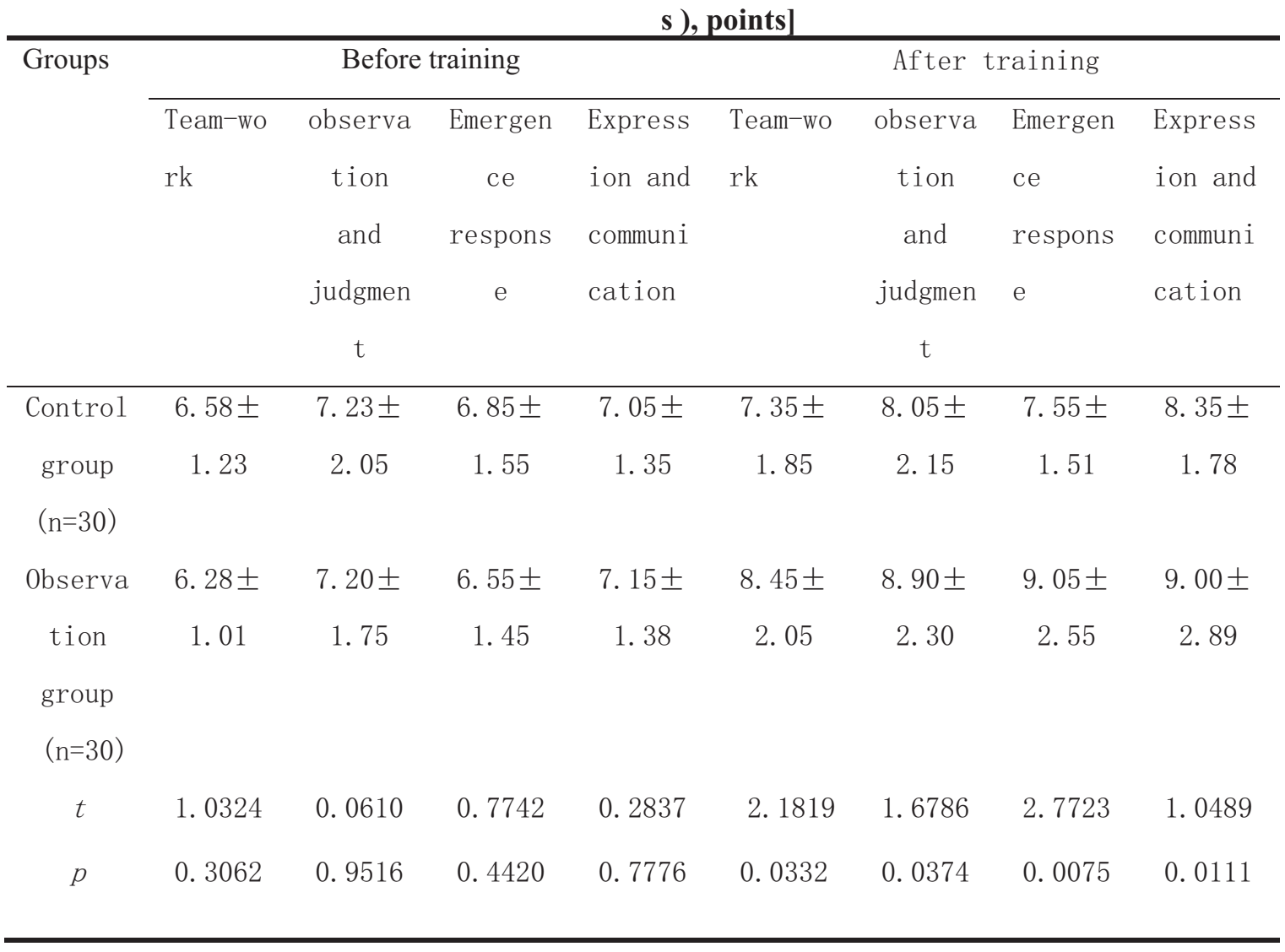


The survey of training satisfaction in two groups of students. Satisfaction of routine nursing training in the control group was $63.33 \%$. Satisfaction of intensive nursing training in the control group was $90.00 \%$, which was higher than that of control group, the comparison had differences $(\mathrm{P}<0.05)$, was statistically significant. Details as shown in table 3 .

Table 3 comparison of satisfaction in different training methods between two groups of [n, \%]

\begin{tabular}{|c|c|c|c|c|c|}
\hline Groups & $\begin{array}{c}\text { Very } \\
\text { satisfied }\end{array}$ & satisfied & $\begin{array}{c}\text { General } \\
\text { satisfied }\end{array}$ & $\begin{array}{c}\text { Not } \\
\text { satisfied }\end{array}$ & $\begin{array}{c}\text { Total } \\
\text { satisfactio } \\
n\end{array}$ \\
\hline $\begin{array}{c}\text { Control } \\
\text { group }(n=30)\end{array}$ & 7 & 12 & 6 & 5 & $19(63.33)$ \\
\hline $\begin{array}{l}\text { Observation } \\
\text { group }(n=30)\end{array}$ & 12 & 15 & 3 & 0 & $27(90.00)$ \\
\hline$u$ & - & - & - & - & 2.3255 \\
\hline$p$ & - & - & - & - & 0.0100 \\
\hline
\end{tabular}

\section{Discussion}

Intensive training refers to a certain degree of learning, using the knowledge to do the short-term, concentrated, high intensity practical training [5], this method allows students to integrate what they have learned to use, allows students to consolidate and promote professional skills. After a period of study, planned, targeted intensive training can reinforce students' basic skills, basic theoretical knowledge and improve students' comprehensive ability in all aspects greatly [6]. Intensive nursing training before internship enables students to operating more standardized, more familiar with the operating procedures, helping to cultivate students into nursing talents with practical ability, laying the foundation to adapt to clinical nursing work[7].

In this study, the control group used conventional nursing training methods and observation group used methods. By comparison, OSCE assessment scores of the two groups of students have improved after training, the observation group has improved significantly, the scores of each aspect after training were higher than the control group's $(\mathrm{P}<0.05)$, which means that planed practicing nursing in intensive nursing training can consolidate students' theory, improve test results; clinical comprehensive ability score of two groups of students after training has improved, the scores of observation group were higher than that of control group $(\mathrm{P}<0.05)$, which means that intensive nursing training can promote the students' teamwork, observation and judgment and other comprehensive ability; Total satisfaction of routine nursing training in control group was $63.33 \%$, total satisfaction of intensive care training in observation group was $90.00 \%$, which was higher than that of the observation group, showing that intensive nursing training can active classroom atmosphere, improve teaching efficiency, and stimulate interest in exercise, improve students' satisfaction [8-9] in training. Clinical nursing teaching practice shows that intensive nursing training can enhance students' skills, cultivate students' teamwork, observation and judgment and other comprehensive ability, it is helpful for students to adapt to clinical nursing in internship as soon as possible, and it's more popular among students [10].

\section{Conclusion}

Intensive nursing skills training can improve students' skills level of operation, clinical comprehensive ability, improve students' satisfaction with clinical nursing teaching. 


\section{References}

[1] Fang Hanping, Liu Hongjuan, ChenYingying, et. Progressive Teaching for Basic Nursing Skills Training for Intern Nursing Students [J]. Journal of Nursing Science, 2016,31 (2): 78-80.

[2] Chen Ying, Wang Huazhi, Ma Yu, et. Reflexive Retrospection and Reflective Diary of Teaching in Clinical Nursing Teaching [J]. Chinese Journal of Contemporary Medicine, 2013,20 (28): 145-147.

[3] Zhong Ling, Deng Hua, Zhao Ruiqian, etc. Strengthen the Basic Nursing Standardization Training and Comprehensively Enhance the Quality of Nursing Services Quality [J]. Journal of Southwest Medical University, 2013,23 (3): 325-326.

[4] Wei Yujing. Effect Evaluation of the Objective Structured Clinical Examination (OSCE). [D]. Shanxi Medical University, 2009.

[5] ALISON E. WHILE. Strengthening the role of the ward manager: a review of the literature [J]. Journal of nursing management, 2014, 22 (6): 685-696.

[6] Yin Hong, Wu Xiaofei. Application of Integrated Scenario Teaching Method in Intensive Nursing Care in Operating Room [J]. Nursing Research, 2014,28 (10): 1265-1266.

[7] Hewko, Sarah J., Cooper, Sarah L., Cummings, Greta G. et al. Strengthening Moral Reasoning Through Dedicated Ethics Training in Dietetic Preparatory Programs [J]. Journal of Nutrition Education and Behavior, 2015, 47 (2): 156 -161.

[8] Tu Wenji, Yang Ping, Pan Hui,etc. Usage and Exploration of Paperless Objective Structured Clinical Examination (OSCE) [J] Basic \& Clinical Medicine, 2015,35 (11): 1576-1578.

[9] Huang Ping, Lu Jianhua, Yao Dingguo etc. Creation and Practice of Chinese Medicine Clinical Skills Training Courses $[\mathrm{J}]$ Zhejiang University of Traditional Chinese Medicine, 2015, 39 (9): 713-714,718.

[10] Gao Jing, Wu Xiaowan, Li Yu, etc. Experiment Teaching of Basic Nursing Skills and Research on Students' Satisfaction of Examination and Evaluation Mechanism [J] Journal of Nursing, 2015, 22 (12): 9-13. 